\title{
Fast fossil rotation of neutron star cores
}

\author{
A. Melatos ${ }^{1}$ \\ amelatos@unimelb.edu.au
}

\begin{abstract}
It is argued that the superfluid core of a neutron star super-rotates relative to the crust, because stratification prevents the core from responding to the electromagnetic braking torque, until the relevant dissipative (viscous or Eddington-Sweet) time-scale, which can exceed $\sim 10^{3} \mathrm{yr}$ and is much longer than the Ekman timescale, has elapsed. Hence, in some young pulsars, the rotation of the core today is a fossil record of its rotation at birth, provided that magnetic crust-core coupling is inhibited, e.g. by buoyancy, field-line topology, or the presence of uncondensed neutral components in the superfluid. Persistent core super-rotation alters our picture of neutron stars in several ways, allowing for magnetic field generation by ongoing dynamo action and enhanced gravitational wave emission from hydrodynamic instabilites.
\end{abstract}

Subject headings: dense matter — hydrodynamics — gravitational waves — stars: interiors - stars: neutron — stars: rotation

\section{Introduction}

Shear flows are endemic to neutron stars. For example, the interpenetrating components of the stellar interior rotate differentially. In the inner crust, an inviscid neutron condensate flows through a rigid nuclear lattice. Nuclear pinning controls the relative velocity (Haskell et al. 2011; Warszawski \& Melatos 2011). The condensate also flows relative to a viscous fluid component, consisting of electrons, protons, and uncondensed neutrons. Entrainment, hydromagnetic stresses, and Ekman pumping control the relative velocity; under certain conditions, the nuclear lattice and viscous component lock together magnetically in $\lesssim 1 \mathrm{~s}$ (Alpar et al. 1984; Jahan-Miri 2002; Sidery \& Alpar 2009;

\footnotetext{
${ }^{1}$ School of Physics, University of Melbourne, Parkville, VIC 3010, Australia
} 
van Eysden \& Melatos 2010). Angular momentum stored in the condensate is released erratically to the crust, causing glitches (Shemar \& Lvne 1996), although the trigger mechanism remains unclear (Warszawski \& Melatos 2008; Melatos \& Warszawski 2009; Sidery et al. 2010; Warszawski \& Melatos 2011).

Additionally, differential rotation can occur between spatially separate regions inside the star. Examples include inductive and capacitive zones, invoked to explain the wide range of post-glitch recovery time-scales in the same pulsar (Alpar et al. 1996; Wong et al. 2001); regions of strong and weak pinning, predicted by nuclear theory (Jones 1991; Avogadro et al. 2007; Haskell et al. 2011); and the crust and core (Peralta et al. 2005, 2006b), especially if the core is a crystalline color superconductor (Alford et al. 2008). Other condensed astronomical bodies are known or suspected to sustain crust-core shear, notably the Earth, where core super-rotation has been observed in seismic travel-time data, and the size of the effect and its variability time-scale are hotly debated (Song \& Richards 1996; Waszek et al. 2011), and the planets Jupiter and Saturn (Guillot 1999).

One natural cause of crust-core shear is gravitational stratification. Oceanographic and other studies of Ekman pumping show that stratification limits the volume of fluid that spins up (down) in an impulsively accelerated (decelerated) container (Walin 1969; Clark 1973; St-Maurice \& Veronis 1975; Abney \& Epstein 1996; Duck \& Foster 2001), at least over the Ekman time-scale. 1 The entire fluid does corotate eventually on a much longer, dissipative time-scale (Greenspan \& Howard 1963; Clark 1973), after inertial oscillations die away. But by then, in a neutron star, the crust has decelerated further electromagnetically, so a crustcore shear always persists. The shear shapes the gravitational wave signal emitted during post-glitch relaxation (van Eysden \& Melatos 2008; Bennett et al. 2010).

In this paper, we apply classic ideas from Solar spin down (Sakurai et al. 1971; Clark 1973) to show that the core of a neutron star rotates systematically faster than the crust in response to the combined action of stratification-limited Ekman pumping and electromagnetic braking. In $\$ 2$, we identify the processes that govern the approach to a steady state in stratified spin down. In \$3, we evaluate the relevant time-scales and estimate the crust-core lag, finding that it is large early in the star's life but decreases with age. In \$4 we ask whether magnetic crust-core coupling is strong enough to erase the crust-core lag and, if so, under what conditions, a subtle question. The observational consequences of a persistent lag for precession, magnetic field evolution, and gravitational radiation are outlined briefly in \$5. Each consequence deserves to be assessed in detail, a challenging task which awaits

\footnotetext{
${ }^{1}$ In this paper, we restrict attention to the spin-down problem relevant to an electromagnetically braking neutron star.
} 
further work.

\section{Stratified Ekman pumping}

The fluid interior of a neutron star responds to changes in the angular velocity $\Omega$ of the crust via Ekman pumping. In an unstratified, Newtonian fluid, Ekman pumping operates as follows: the Coriolis force drives a secondary circulation in boundary layers of thickness $E^{1 / 2} R$ abutting the crust; then the boundary flow sucks fluid from the interior, spins it down, and recycles it on the Ekman time-scale $t_{E}=E^{-1 / 2} \Omega^{-1}$ (Greenspan \& Howard 1963). The Ekman number, $E=\nu /\left(R^{2} \Omega\right)$, is a function of the kinematic viscosity $\nu$ and stellar radius $R$. It is the only dimensionless variable in the problem. In an unstratified, twocomponent, Hall-Vinen-Bekharevich-Khalatnikov superfluid, much the same thing happens to the viscous component, which in turn drags along the inviscid component via mutual friction (van Eysden \& Melatos 2010, 2011, 2012).

Stratification alters Ekman pumping fundamentally. In addition to $E$, two new dimensionless variables enter the problem: the Burger number, $S=N^{2} / \Omega^{2}$, which quantifies the relative strength of the buoyancy and Coriolis forces, and the Prandtl number, $\sigma=\nu / \kappa$, which quantifies the relative rate at which momentum and heat are transported diffusively. The symbols $N$ and $\kappa$ stand for the Brunt-Väisälä frequency and thermal diffusivity respectively. We see below that, in a neutron star, $S$ is large [cf. Baym et al. (1976)], and $\sigma$ is either small or of order unity, depending on exactly how superfluidity affects $\kappa$. The interplay and ordering of E, S, and $\sigma$ lead to a rich variety of motions (Sakurai et al. 1971; Clark 1973; St-Maurice \& Veronis 1975; Spence et al. 1992).

First and foremost, stratification limits the interior volume influenced by Ekman pumping to a boundary region, called a buoyancy layer, of thickness $S^{-1 / 2} R$ (Walin 1969; Clark 1973). In a neutron star, the buoyancy layer is thicker than the unstratified Ekman layer $\left(S \ll E^{-1}\right)$, but it does not suck in, spin down, and recycle interior fluid to produce uniform rotation. Instead, on the Ekman-like time-scale $S^{-1 / 2} E^{-1 / 2} \Omega^{-1}$, the interior organizes itself into a quasi-steady, sheared, azimuthal flow, whose angular velocity runs smoothly from its original value deep in the core to match the spun-down buoyancy layer at the crust (Sakurai et al. 1971; Spence et al. 1992). The angular velocity profile is set by the geometry and temperature boundary conditions; in general, Taylor-Proudman columnarity breaks down (St-Maurice \& Veronis 1975; Friedlander 1976).

Secondly, stratification distorts the boundary layer (Walin 1969). In a cylinder, the side wall cannot carry a vertical (i.e. falling) mass flux, so it cannot accept an $O\left(E^{1 / 2}\right)$ Ekman 
inflow. As a result, fluid is pushed into the corners and thence erupts into the interior as a jet, leaving behind a stagnant region (Spence et al. 1992). Eruption also occurs in a sphere, with Ekman suction turning into blowing at latitudes $\lesssim 24^{\circ}$ (Clark et al. 1971; Friedlander 1976). The jet exists for $S \sigma>E^{2 / 3}$, which always holds in a neutron star.

Ultimately, of course, the sheared interior flow must evolve towards uniform rotation. When does this happen? Not until the faster of two dissipative processes takes hold: viscous diffusion (viscous time-scale $t_{\nu}=E^{-1} \Omega^{-1}$ ), or thermally driven circulation (Eddington-Sweet time-scale $t_{\kappa}=\sigma S E^{-1} \Omega^{-1}$, i.e. the thermal diffusion time-scale multiplied by $S$ ) (Clark 1973; St-Maurice \& Veronis 1975; Hollerbach 2009). If thermal conduction in the neutron superfluid is mainly collisional, one has $\sigma \sim 1$ (Baiko et al. 2001; Urpin 2003), and it is safe to ignore the latter process. However, in terrestrial superfluids like helium II, convective counterflow of the small fraction of uncondensed neutrons boosts the effective thermal conductivity dramatically (Cutler \& Lindblom 1987; Baiko et al. 2001). The possibility that similar physics is at work in a neutron star is hard to quantify but cannot be dismissed, so we analyse it too in what follows. For $\sigma S<1$, a thermal layer diffuses inwards, its thickness growing with time $t$ as $(\kappa t)^{1 / 2}$. It encounters the inner edge of the buoyancy layer at $t=\sigma S^{-1} E^{-1} \Omega$, whereupon the angular velocity decrement imposed by the crust diffuses inwards within a layer whose thickness grows $\propto t^{1 / 4}$, reaching the center once $t$ equals $t_{\kappa}$ (Sakurai et al. 1971; Clark 1973; St-Maurice \& Veronis 1975).

\section{Long-term crust-core shear}

The maximum angular velocity gradient within the star depends on where the electromagnetic braking time-scale $\Omega / \dot{\Omega}$ fits into the hierarchy of hydrodynamic time-scales. Over the long term, there are two astrophysically interesting scenarios: $t_{E}<\Omega / \dot{\Omega}<\min \left(t_{\nu}, t_{\kappa}\right)$ and $\min \left(t_{\nu}, t_{\kappa}\right)<\Omega / \dot{\Omega}$. In the first scenario, early in the star's life, the angular velocity gradient equals $\left(\Omega_{0}-\Omega\right) / R$ (if $\left.t_{\nu}<t_{\kappa}\right)$ or $\left(\Omega_{0}-\Omega\right)\left(t / t_{\kappa}\right)^{1 / 4} / R$ (if $t_{\nu}>t_{\kappa}$ ), where $t$ is the star's age, and $\Omega_{0}$ is the initial angular velocity; in other words, the rotation of the core is a fossil record of its rotation at birth. In the second scenario, when the star is older, the angular velocity gradient equals $|\dot{\Omega}| \min \left(t_{\nu}, t_{\kappa}\right) / R$; all memory of the natal rotation disappears, and the gradient is proportional to the small amount of spin down that occurs while the relevant dissipative process unfolds [see $\S 6$ in Sakurai et al. (1971) and $\S 5$ in Clark (1973)]. 2

\footnotetext{
${ }^{2}$ For $\sigma S \ll 1$, Friedlander (1976) found that uniform rotation is established on the viscous time-scale $t_{\nu}$, if the crust experiences a constant spin-down torque, contrasting with Sakurai et al. (1971), who found that $t_{\kappa}$ is the corotation time-scale, if the crust decelerates impulsively. This subtlety is an idiosyncracy of the
} 
To quantify the shear, we refer to the latest, ab initio calculations of transport coefficients in bulk nuclear matter (Yakovlev et al. 1999; Baiko et al. 2001; Shternin \& Yakovlev 2008) to evaluate the dimensionless hydrodynamic variables for a standard neutron star with mass $\approx 1.4 M_{\odot}$ and $R=10^{6} \mathrm{~cm}:$

$$
\begin{aligned}
E & =1.4 \times 10^{-10} R_{\nu}\left(\frac{\rho}{\rho_{0}}\right)\left(\frac{T}{10^{8} \mathrm{~K}}\right)^{-5 / 3}\left(\frac{\Omega}{10^{2} \mathrm{~s}^{-1}}\right)^{-1} \\
S & =2.9 \times 10^{2}\left(\frac{\rho}{\rho_{0}}\right)^{1 / 3}\left(\frac{\Omega}{10^{2} \mathrm{~s}^{-1}}\right)^{-2}, \\
\sigma & =7.4\left(\frac{R_{\nu}}{R_{\kappa}}\right)\left(\frac{\rho}{\rho_{0}}\right)^{1 / 3}\left(\frac{T}{10^{8} \mathrm{~K}}\right)^{1 / 3} .
\end{aligned}
$$

In equations (11)-(3), $\rho$ and $T$ denote the density and temperature in the core, $\rho_{0}=2.8 \times$ $10^{14} \mathrm{~g} \mathrm{~cm}^{-3}$ is the nuclear saturation density, and $R_{\nu}$ and $R_{\kappa}$ are superfluidity suppression factors for $\nu$ and $\kappa$ respectively. 34 We assume npe matter for simplicity: the electrons are ideal, degenerate, and ultrarelativistic, while the nucleons are nonideal and nonrelativistic (Yakovlev et al. 1999). To calculate the thermal conductivity, we assume that neutronneutron and neutron-proton quasiparticle collisions dominate (Baiko et al. 2001) (but set the dressed masses equal to the bare masses for simplicity), neglect electron conduction, neglect superfluidity in the scattering frequencies (but not elsewhere), and calibrate the result against Figure 1 in the latter reference. To calculate the shear viscosity, we assume that electronelectron collisions dominate, except at $T \sim 10^{7} \mathrm{~K}$ where neutrons also contribute, we neglect muons (consistent with the npe assumption), and we include the important effect of Landau damping mediated by weakly screened transverse plasmons (Shternin \& Yakovlev 2008). Calibrating against Figure 4 in the latter reference, we see that Landau damping lowers $\nu$ by nearly an order of magnitude with respect to the traditional value (Cutler \& Lindblom 1987), and $\nu$ scales as $T^{-5 / 3}$ instead of $T^{-2}$, which is astrophysically significant because it

regime $\sigma S \ll 1$, which is unlikely to be reached in a neutron star, even if superfluidity enhances $\kappa$.

${ }^{3}$ In general, $R_{\nu}$ and $R_{\kappa}$ are complicated functions of $T$, with $R_{\nu} \neq R_{\kappa}$. As a rough rule of thumb, one finds $R_{\nu}=R_{\kappa}=1$ in the absence of superfluidity, $R_{\nu} \approx R_{\kappa} \approx 0.2$ if only the neutrons are strongly superfluid, and $R_{\nu} \approx R_{\kappa} \approx 0.05$ if both the neutrons and protons are strongly superfluid. If convective counterflow in the uncondensed neutrons facilitates thermal transport, the foregoing approximations still apply to $R_{\nu}$, and we have $R_{\kappa} \gg R_{\nu}$, but the value of $R_{\kappa} / R_{\nu}$ is not known accurately (Yakovlev et al. 1999; Baiko et al. 2001; Shternin \& Yakovlev 2008).

4 The core temperature is related to the surface temperature via a two-zone heat-blanket model (Gudmundsson et al. 1982), and the surface temperature is related to the age $t$ via 'fast' (superfluid) cooling curves (including kaon and pion condensates and the direct Urca process) calibrated against 11 neutron stars with measured thermal X-ray emission [see Figure 2 in Page (1998)]. 
leaves a $T$ dependence in $\sigma$. The Brunt-Väisälä frequency, arising from departures from beta equilibrium, is proportional to the square root of the electron-neutron ratio and is taken from Reisenegger \& Goldreich (1992).

Figure 1 illustrates how much core super-rotation to expect in neutron stars of different ages. The results are expressed in terms of the Rossby number, Ro $=\Delta \Omega / \Omega$, where $\Delta \Omega$ is the angular velocity of the core relative to the crust. One obtains $R_{0}=\Omega_{0} / \Omega-1$ and Ro $=|\dot{\Omega}| \min \left(t_{\nu}, t_{\kappa}\right) / \Omega$ respectively in the two scenarios described at the start of 93 , Contours of Ro are graphed on the plane spanned by the rotation period $P$ and its time derivative $\dot{P}$. Known radio pulsars are overplotted as blue dots. The blue band at the left of the diagram marks the excluded region $S \lesssim 1$, where stratification is weak, buoyancy does not impede Ekman pumping, there is no fossil rotation, the long-term shear Ro $\approx t_{E} / t \lesssim 10^{-6}$ is too small to be of interest, and the analysis in this paper does not apply.

Consider first the example $R_{\nu}=R_{\kappa}=0.05$ (red, dashed contours), which corresponds to strong superfluidity in the neutrons and protons, without counterflow-enhanced thermal conduction. In this regime, we find $t_{\nu}<t_{\kappa}$; viscous diffusion pre-empts thermally driven circulation throughout the displayed subset of the $P-\dot{P}$ plane, and the orange shading in the figure (explained in the next paragraph) is irrelevant. In most of the figure, below and to the right of the green shading, we also have $t>t_{\nu}$ and hence $\operatorname{Ro}=t_{\nu} /(2 t)$. The red contours run from $\mathrm{Ro}=10^{-1}$ to $10^{-6}$, spaced by one dex. Numerous objects with spin-down ages $\lesssim 10^{4}$ yr are predicted to have relatively high Rossby numbers. In particular, there are three objects with Ro $\geq 10^{-1}$ (the young pulsar PSR J1846-0258 in the supernova remnant Kesteven 75, and the magnetars PSR J1550-5418 and PSR J1907+0919), and 18 with $10^{-2} \leq \mathrm{Ro}_{\mathrm{S}} \leq 10^{-1}$ (including three with Ro $\approx 0.1$ ). PSR J1846-0258, which is only $0.7 \mathrm{kyr}$ old, and whose braking index has been measured by absolute pulse numbering, is an especially good rotation fossil candidate (Gotthelf et al. 2000; Livingstone et al. 2006). In the top left corner of the figure, outlined by the green triangle, where young pulsars with $t \lesssim 10^{3}$ yr and $T \gtrsim 10^{8} \mathrm{~K}$ reside, we find $t<t_{\nu}$ and hence predict $\mathrm{Ro} \gtrsim 1$, i.e. the core retains much of its natal angular velocity. No radio pulsars have been discovered in the green triangle to date, but such objects, whether young like PSR J1846-0258 or magnetar-like, are easily accommodated within our current picture of neutron star evolution.

Next consider the example $R_{\nu}=0.05, R_{\kappa}=5 \times 10^{2}$ (black, unbroken contours), which models roughly the counterflow effect. The orange shading now becomes relevant; it divides the $P-\dot{P}$ plane into two parts. In the white region to the right of the orange curve, we find $t_{\nu}<t_{\kappa}$, and nothing changes from the previous example: viscous diffusion dominates, and the black Ro contours overlap the red ones. To the left of the orange curve, however, the opposite is true: we find $t_{\kappa}<t_{\nu}$, and thermally induced circulation occurs faster than 


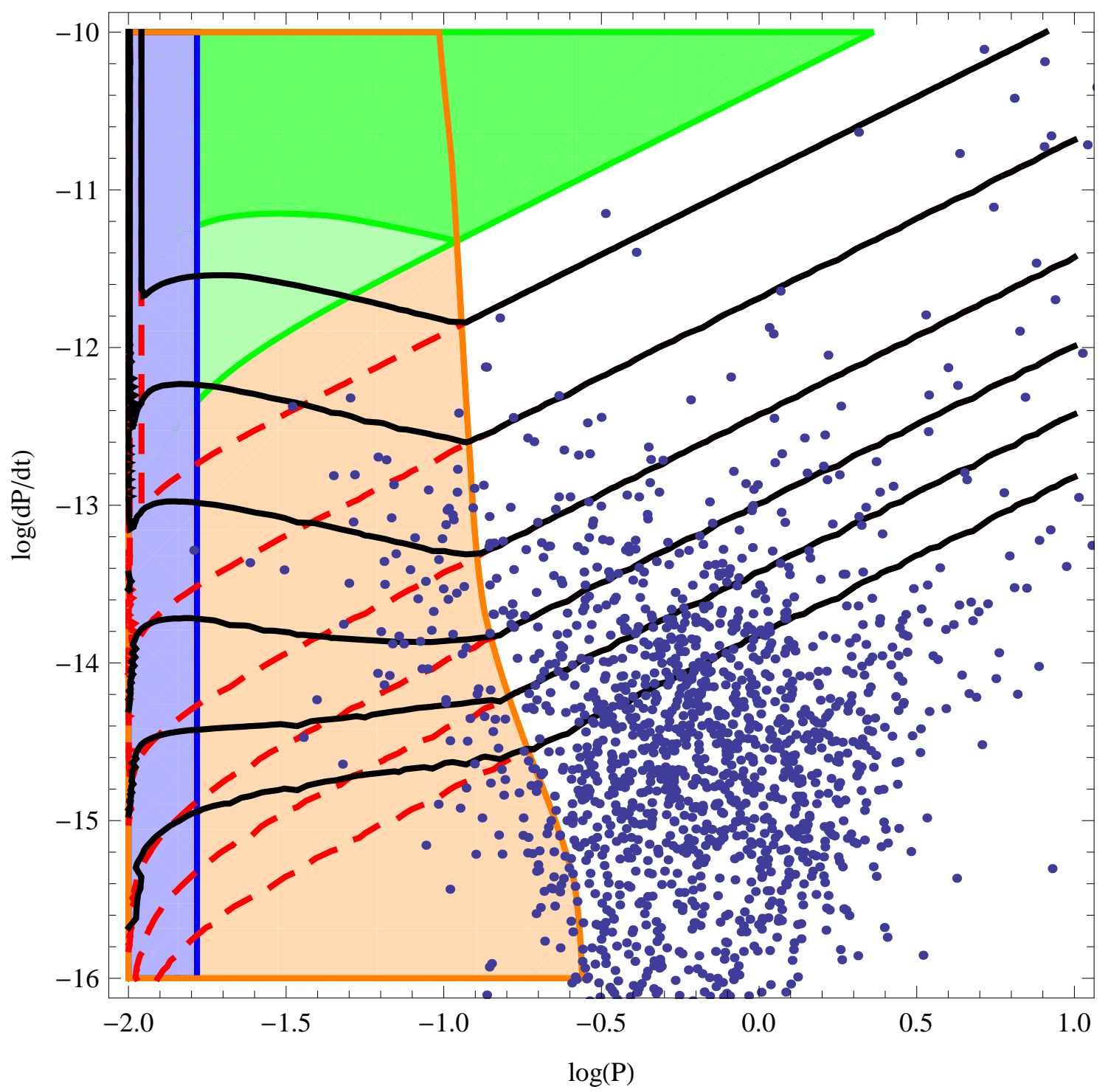

Fig. 1.- Contour plot of Rossby number as a function of spin period $P$ (horizontal $\log$ axis, in s) and period derivative $\dot{P}$ (vertical $\log$ axis, in $\mathrm{s} \mathrm{s}^{-1}$ ), running from $\mathrm{Ro}=0.1$ (top contour) to $10^{-6}$ (bottom contour) in steps of 1 dex. Red, dashed curves: strong neutron and proton superfluidity, collisional thermal conduction $\left(R_{\nu}=R_{\kappa}=0.05\right)$. Black, unbroken curves: counterflow-enhanced thermal conduction $\left(R_{\nu}=0.05, R_{\kappa}=5 \times 10^{2}\right)$. Blue dots mark known radio pulsars. The colored regions cover the following regimes. Blue: $S<20$, stratification too weak to impede Ekman pumping, Ro negligible. Heavy green: fossil stage, $t<t_{\nu}<t_{\kappa}$. Light green: fossil stage, $t<t_{\nu}<t_{\kappa}\left(t<t_{\kappa}<t_{\nu}\right)$ for $R_{\kappa}=0.05\left(5 \times 10^{2}\right)$. Orange: $t>\min \left(t_{\nu}, t_{\kappa}\right)=t_{\kappa}$, natal rotation erased, Ro decreases monotonically with age, thermally driven circulation pre-empts viscous diffusion. White: $t>\min \left(t_{\nu}, t_{\kappa}\right)=t_{\nu}$, viscous diffusion pre-empts thermally driven circulation. Other parameters: $\rho=\rho_{0}, \Omega_{0}=200 \pi \mathrm{s}^{-1}$ (fiducial), $T$ from Page (1998) (see footnote 4). 
viscous diffusion. Below the irregularly shaped, heavy green region, we also have $t>t_{\kappa}=$ $9.7\left(R_{\kappa} / 5 \times 10^{2}\right)^{-1}\left(\rho / \rho_{0}\right)^{-1 / 3}\left(T / 10^{8} \mathrm{~K}\right)^{2}\left(\Omega / 10^{2} \mathrm{~s}^{-1}\right)^{-2} \mathrm{yr}$ and hence Ro $=t_{\kappa} /(2 t)$. That is, when $R_{\kappa}$ is large, the green region to the right of the orange curve $\left(t_{\nu}<t_{\kappa}\right)$ is a promising hunting ground for rotation fossils with $\mathrm{Ro}_{2} \geq 1$; we do not expect to find many to the left of the orange curve. Likewise, when $R_{\kappa}$ is large, it is harder to find objects with Ro less than unity but still relatively large; as the black contours tilt upwards in the orange region, one needs a younger, faster pulsar to achieve a given Ro than when $R_{\kappa}$ is small, yet young pulsars spin down quickly. A meaningful fraction of the pulsar population lies within the orange region. No known objects are predicted to have $R_{0} \geq 0.1$, three have $10^{-2} \leq$ Ro $\leq 10^{-1}$ [the gamma-ray pulsar PSR J1023-5746, discovered in a blind Fermi Large Area Telescope search (Saz Parkinson 2010), PSR J0540-6919 in the Large Magellanic Cloud, and the Crab], and 16 have $10^{-3} \leq$ Ro $\leq 10^{-2}$

Figure 1 also maps the history of differential rotation in individual objects. As a pulsar brakes electromagnetically, it moves from the top left to the bottom right of the figure along a diagonal spin-down trajectory, $P \dot{P}=$ constant. If the trajectory intersects the green triangle, the early part of the star's life is spent as a rotation fossil. 5 When the star crosses from the green region into the orange or unshaded regions, Ro drops suddenly on the time-scale $\min \left(t_{\nu}, t_{\kappa}\right)$, as dissipative processes take over. Subsequently, the crust-core shear decreases monotonically as the star brakes and cools, with $\dot{\Omega} / \Omega \propto t^{-1}$ and hence Ro $\propto t^{-1} T^{5 / 3}$ or Ro $\propto T^{2}$ in the unshaded or orange regions respectively. 6 If the trajectory starts outside the green triangle, the latter behavior characterizes the star's whole life: there is no fossil stage. A wide range of terminal Ro values are possible, when the trajectory intersects the pair-cascade death line (Hibschman \& Arons 2001).

\section{Magnetic coupling}

How does the stellar magnetic field alter the spin-down scenario above? This question turns out to be subtle. The physics depends sensitively on the internal magnetic topology and the multi-component nature of the superfluid interior, both of which remain highly uncertain. A proper understanding also requires a theory of Ekman pumping in a stratified, magnetized fluid in a sphere, which does not exist yet in the literature even for uniform magnetization, let alone for a realistic (e.g. linked poloidal-toroidal) field geometry or for a

\footnotetext{
${ }^{5}$ For the minority of neutron stars born in the blue region, where stratification is weak, Ekman pumping erases the natal rotation on the fast time-scale $t_{E}$.

6 The transition between the two scalings occurs smoothly at the wavy orange curve in Figure 1.
} 
superconductor. In this section, therefore, we restrict ourselves to canvassing some of the main issues and presenting associated order-of-magnitude scalings, noting that large-scale numerical simulations will ultimately be required to settle the matter decisively.

Magnetic coupling in its simplest guise (i.e. in an unstratified, uniformly magnetized star) speeds up angular momentum transport dramatically, enforcing corotation between the crust and core over $\sim 10^{2}$ s for typical magnetic field strengths and spins. The process occurs in two stages. Firstly, the charged component of the superfluid is accelerated by Lorentz forces, mediated not by Alfvén waves but rather by rotation-dominated, hydromagneticinertial waves, if the star is not superconducting (Easson 1979b), or by cyclotron-vortex waves, whose restoring force comes from the vortex tension, if the star is superconducting (Mendell 1998). Secondly, the charged fluid drags along the neutron condensate via mutual friction, as charges scatter off neutron vortices magnetized by the Fermi liquid interaction (entrainment) (Alpar et al. 1984). Overall, for uniform magnetization, the coupling timescale is given by $t_{B}=2\left(V_{A} / R \Omega\right)^{-2 / 3} \Omega^{-1}=6.6\left(\rho / \rho_{0}\right)^{1 / 3}\left(\Omega / 10^{2} \mathrm{~s}^{-1}\right)^{-1 / 3}\left(B / 10^{12} \mathrm{G}\right)^{-2 / 3} \mathrm{~s}$ in a non-superconducting star (Easson 1979b), where $V_{A}=\left(B^{2} / 4 \pi \rho\right)^{1 / 2}$ is the Alfvén speed, and $B$ denotes the magnetic field strength. In a type II superconductor, $B^{2}$ is replaced by $B H_{c 1}$ in the formula for $t_{B}$, where $H_{\mathrm{c} 1} \sim 10^{15} \mathrm{G}$ is the lower critical field, strengthening the coupling further.

In a stratified star, the magnetized Ekman problem remains unsolved. Broadly, though, stratification (and compressibility) inhibit crust-core coupling, just as they do in an unmagnetized star (Abney \& Epstein 1996). To estimate the stratified magnetic coupling time-scale, we follow the pioneering order-of-magnitude analysis in $\S 6$ of Mendell (1998), which is arguably somewhat representative even though it applies to the idealized case of uniform magnetization. Mendell (1998) showed that the $t_{B}$ formula in the previous paragraph applies only to the uppermost, buoyancy-limited boundary layer adjacent to the crust. One then imagines a stack of $\approx R \Omega / V_{A}$ buoyancy-limited layers reaching from the crust to the core, which are activated sequentially and combine to give a revised time-scale, $t_{B}=2\left(V_{A} / R \Omega\right)^{-5 / 3} \Omega^{-1}=3.9 \times 10^{4}\left(\rho / \rho_{0}\right)^{5 / 6}\left(\Omega / 10^{2} \mathrm{~s}^{-1}\right)^{2 / 3}\left(B / 10^{12} \mathrm{G}\right)^{-5 / 3} \mathrm{~s}$. Hence it is possible to have $t_{B}>t_{\nu}$ - and hence maintain core super-rotation on the viscous time-scale, as in $\oint_{2}$ and $\$ 3$ - in objects with moderately magnetized interiors and/or fast spins, satisfying $\left(B / 10^{12} \mathrm{G}\right)^{5 / 3}\left(\Omega / 10^{2} \mathrm{~s}^{-1}\right)^{-2 / 3}<5.5 \times 10^{-4} R_{\nu}\left(\rho / \rho_{0}\right)^{11 / 6}\left(T / 10^{8} \mathrm{~K}\right)^{-5 / 3}$. Such objects may be found squarely within the main pulsar population in Figure 1 (e.g. $B \lesssim 10^{10} \mathrm{G}$ ) or among the recycled millisecond pulsars, provided that the internal fields in the latter are actually reduced rather than just buried, cf. Konar \& Bhattacharya (1997) and Payne \& Melatos (2004). The above conclusions are consistent with related work on boundary-layer damping of r-modes. In unmagnetized r-modes, it is found that compressibility approximately halves the damping rate, while stratification has almost no effect, because the radial fluid velocity 
component in an r-mode is tiny (Glampedakis \& Andersson 2006), unlike in the spin-down problem considered here, where the radial and angular velocity components are of the same order and stratification matters (Abney \& Epstein 1996). In magnetized r-modes, analyzed for the idealized, unstratified problem of a radial magnetic field with uniform or cosinelatitude magnitude, Lorentz forces shorten the damping time-scale, offset partially by the superfluid mutual friction, because damping occurs in a boundary layer abutting the crust, where buoyancy is negligible (Mendell 2001; Kinney \& Mendell 2003), unlike in core spin down.

In a stratified star with a realistic internal magnetic geometry, things are more uncertain. Arguably, however, the overall impact of a nontrivial geometry is to lengthen the magnetic coupling time-scale further. One can see this qualitatively by considering the dispersion relations of hydromagnetic waves in a stratified slab, where the stratification axis $\mathbf{n}$ is inclined obliquely to both the background magnetic field $\mathbf{B}_{0}$ and the direction $\mathbf{k}$ of wave propagation. A thorough treatment of this problem in plane-parallel geometry (without rotation, unfortunately) is given for various wave modes in Chapter 7 of Goedbloed \& Poedts (2004). The details fall outside the scope of this paper, but generically one discovers that the phase speed is typically lower than the buoyancy-limited Alfvén speed in a uniform magnetic field and is much lower for certain orientations. For example, when $\mathbf{n}$ is perpendicular to $\mathbf{k}$ and $\mathbf{B}_{0}$, and the wave propagates obliquely with respect to $\mathbf{B}_{0}$, the phase speeds of the Alfvén and slow modes (which are favored because they displace fluid horizontally, unlike the fast mode) tend to zero in the small-beta (pressure-dominated) regime relevant to neutron stars, as illustrated in Figure 7.10 of Goedbloed \& Poedts (2004). In the uniform-B $\mathbf{B}_{0}$ calculations attempted so far in the neutron star literature, such orientations are avoided by construction but they are inevitable in realistic geometries. For example, in a linked poloidal-toroidal structure (Braithwaite \& Nordlund 2006; Lander et al. 2012), where field loops close inside the star, many internal surfaces exist where unfavorable orientations of $\mathbf{n}, \mathbf{k}$, and $\mathbf{B}_{0}$ are achieved, and buoyancy-modified hydromagnetic waves slow down dramatically, lengthening the crust-core magnetic coupling time. In a tangled field, this is even more likely to occur (Braithwaite \& Nordlund 2006). Goedbloed \& Poedts (2004) emphasized in $\$ 7.3 .3$ of their book that these subtle effects emerge fully when oblique propagation $\left(0<\left|\hat{\mathbf{k}} \cdot \hat{\mathbf{B}}_{0}\right|<1\right)$ is considered, and that they are expected to be even more prevalent when $\mathbf{n}$ is inclined obliquely to $\mathbf{B}_{0}$ as well, the relevant scenario in general in a neutron star [and outside the scope of Goedbloed \& Poedts (2004)]. There is no substitute for a self-consistent, three-dimensional numerical simulation to quantify these difficult matters. The prospects are fair that suitable codes and computational platforms will become available shortly that are equal to the task.

In a realistic magnetic geometry, another crucial complication arises: many field topologies are incapable of enforcing crust-core corotation at all, no matter how fast the magnetic 
coupling. Quoting from Easson (1979a), who demonstrated this counter-intuitive property analytically, "the field must have a topology such that the equation for the perturbation $\mathbf{B}^{\prime}$ in the field induced by the slowing down has nonsingular, single-valued solutions". At first blush, this sounds like a side-issue of purely academic interest, but nothing could be further from the truth. There exist at least two important topologies (which are popular in the literature, incidentally) that violate the above condition and cannot enforce corotation: (1) axisymmetric, poloidal fields with loops closing inside the star; and (2) axisymmetric, toroidal fields. To see why, consider the momentum equation in superfluid magnetohydrodynamics, viz. equation (1) in Easson (1979a). Several of the forces are not conservative in general, e.g. the Lorentz force and the Fermi liquid interaction. 7 The nonconservative forces cannot be balanced everywhere by pressure gradients and buoyancy, so $\mathbf{B}^{\prime}$ tries to adjust until a balance is achieved. But often there is no way to do so. For example, if $\mathbf{B}_{0}$ is poloidal and axisymmetric, then $\mathbf{B}^{\prime}$ is toroidal and the toroidal $(\varphi-)$ component of the momentum equation reduces to $(4 \pi)^{-1}\left(\mathbf{B}_{0} \cdot \nabla\right)\left(\varpi B_{\varphi}^{\prime}\right)=F(\mathbf{q}, \varpi)$ in equilibrium, where $\varpi$ denotes the cylindrical radius, and the right-hand side is a complicated nonconservative function $F(\ldots)$ of unperturbed variables $\mathbf{q}$ like the neutron and proton mass densities, viz. equation (6) in Easson (1979a). Integrating along a poloidal field line that closes inside the star, one reaches the unphysical conclusion that $\varpi B_{\varphi}^{\prime}$ is multi-valued. It is tempting to presume that the plasma can always find some way to adjust slightly and quasi-statically near a sensible topology to fix the problem, "so that the relevant integrals along all closed loops give zero" (Easson 1979a). But Easson (1979a) proved directly from the hydromagnetic equations of motion that the adjustment fails to achieve balance except in very special situations, because $F(\mathbf{q}, \varpi)$ is a function of variables that depend only on the unperturbed state of the star (density, temperature, $\mathbf{B}_{0}$, and the crust angular velocity, which do not respond as $\mathbf{B}^{\prime}$ adjusts) as well as the angular velocity of the superfluid vortex array, which is governed by a superfluid equation of motion independent of $\mathbf{B}^{\prime}$. These important points are not appreciated widely in the literature and deserve careful study in future. For example, is the set of fields $\mathbf{B}_{0}$ that give unphysical solutions for B' "large" or "small"? Easson (1979a) expressed a hope that numerical simulations would answer this question, but the problem is formidable, and the relevant simulations have not yet been done.

Magnetic flux is concentrated into quantized flux tubes in a type II superconductor, modifying the magnetohydrodynamic equations of motion (Glampedakis et al. 2011). The

\footnotetext{
7 The entrainment force looks superficially like it is constructed out of gradients of the internal energy and proton effective mass, but the prefactors multiplying these gradients, like the proton number density and pressure, are functions of position even in equilibrium due to compositional stratification; see equation (2) in Easson (1979a).
} 
implications for core super-rotation remain highly uncertain. One outcome is to replace $B^{2}$ by $B H_{\mathrm{c} 1}$ in the classic formula for the magnetic coupling time-scale, as described at the start of this section, but even this result has not yet been confirmed in realistic geometries and in the presence of stratification, where other length-scales and directional effects may enter the problem. Another outcome is that the flux tubes are expected to interact with the superfluid vortices in the neutron condensate, which are magnetized by entrainment. Opinions remain divided as to whether the interaction is strong, locking together the charged and neutral components (Ruderman et al. 1998), or weak, as in the recent "snowplow" model of glitches (Haskell et al. 2012; Seveso et al. 2012), if the core is a type I superconductor (Jones 2006), contains hyperons (Babaev 2009), or is the seat of vortex instabilities (Peralta et al. 2006b; Andersson et al. 2007; Link 2012). We do not attempt to adjudicate these difficult matters in this paper. Efforts are currently under way to calculate analytically the Ekman coupling time-scale in a type II superconductor including some of the above physics, albeit in an idealized plane-parallel geometry and a uniform magnetic field. 8

Whether strong or weak, magnetic coupling does not engage the uncondensed neutrons, which are viscous, participate in Ekman pumping, carry inertia, and therefore play a role in maintaining core super-rotation [and in explaining radio timing data of pulsar glitch recoveries; see van Eysden \& Melatos (2010)]. It is sometimes assumed that the uncondensed neutrons carry a tiny fraction of the star's inertia, because their density is proportional to $\exp \left(-\Delta / k_{\mathrm{B}} T\right)$ in the ideal Bardeen-Cooper-Schrieffer theory, and $k_{\mathrm{B}} T$ is much smaller than the superfluid gap $\Delta$ for most neutron pairing schemes (Yakovlev et al. 1999). However, the ideal exponential gap formula tells only part of the story. Applied to superfluid helium, it predicts zero uncondensed atoms as $T \rightarrow 0$, whereas the experimentally measured fraction is $\approx 14 \%$ due to van der Waals forces. It is likely that the nuclear color force is nonideal in the same way, as the quarks and gluons inside nucleons are polarized by random motions of nearby nucleons at high densities (Weber 2005). The effect has not been quantified yet from first principles, as it requires a hard calculation in quantum chromodynamics, but there are good indications that a van der Waals equation of state is consistent with measurements of the nuclear liquid-gas transition (Torrieri \& Mishustin 2010) and also with scattering experiments (Kaiser et al. 1997). If the uncondensed fraction is substantial, it helps maintain residual core super-rotation via slow, unmagnetized, stratification-limited Ekman pumping, even when the other stellar components couple magnetically on a fast time-scale. 9

\footnotetext{
${ }^{8}$ K. Glampedakis, private communication.

9 Recently an analytic theory of unmagnetized superfluid spin down has been developed in arbitrary geometry which self-consistently incorporates Ekman pumping, mutual friction, and the back reaction of the fluid on its container. The theory reproduces the results of classic laboratory experiments on liquid
} 


\section{Observational signatures}

How might one observe a crust-core shear of the magnitude in Figure 1]? The question is too multi-faceted to answer properly all at once, but we tender a few suggestions. Taking inspiration from the Earth, where seismic data carry the imprint of the super-rotating core (Song \& Richards 1996; Zhang et al. 2005; Waszek et al. 2011), one option is to look for rotational splitting of the quasi-periodic oscillations observed in the X-ray light curve in the immediate aftermath of a magnetar giant flare (Strohmayer \& Watts 2006). At present, there is a lively debate as to whether magnetar oscillations arise from shear modes in the crust, magneto-inertial modes in the core, a combination of the two, or something else entirely (Lander et al. 2010). Theoretical treatments invariably start by perturbing a uniformly rotating background structure, and the rotational splitting is assumed to be small, as magnetars are slow rotators. However, in objects where at least some of the natal rotation of the core is preserved as in Figure 1, one might hope to see greater rotational splitting.

Another possible signature is precession. A body composed of a rigid crust and liquid mantle precesses in a superposition of two modes (four if it also contains a solid inner core) excited by differential rotation, namely a Chandler wobble and free-core nutation (Kitiashvili \& Gusev 2008a); think of an egg, whose yolk rotates differentially. The mode frequencies are related to the inertia of the components and the strength of crust-core coupling via magneto-inertial (Baym et al. 1976) or elasto-inertial (Bastrukov et al. 1999) waves. The model can be matched to the harmonics observed in PSR B1828-11 (Stairs et al. 2000; Kitiashvili \& Gusev 2008a) 10 and to similar periodic phenomena ranging from months to years in other objects (Kitiashvili \& Gusev 2008b) to infer the crust-core shear in principle. A long-period crust-mantle wobble may also be excited by a rotational glitch, when a portion of the crust-core shear is impulsively reset (Yuan et al. 2010).

If verified observationally, core super-rotation enriches neutron star astrophysics in two important ways. First, it implies ongoing, self-limiting, toroidal magnetic field generation. One might argue that magnetic stresses should react to erase the shear on the time-scale

helium (Tsakadze \& Tsakadze 1980) to $0.5 \%$ accuracy with zero free parameters van Evsden \& Melatos 2011), yields excellent fits to Vela's quasi-exponential glitch recoveries, and predicts without adjustment the "overshoot" observed in the Crab (van Eysden \& Melatos 2010), all with zero magnetic coupling. Of course, nonzero magnetic coupling is also consistent with the glitch data, provided that vortex pinning in the inner crust is invoked to retard the response; one is then led to postulate multiple pinning zones to match the multiple recovery time-scales observed in individual objects (Alpar et al. 1984).

10 The oscillatory timing residuals and pulse profile changes in PSR B1828-11 are now interpreted in terms of switching between two magnetospheric states (Lyne et al. 2010). 
$\mathrm{Ro}^{-1} \Omega^{-1}$, but the Sun provides a clear counter-example. Many resistive and other processes intervene to pinch off toroidal magnetic flux tubes before the back-reaction becomes too strong, e.g. the current-induced Tayler instability, which is especially active for high magnetic Prandtl numbers (and is modified by the Hall effect in magnetars) (Rüdiger et al. 2009), or magnetic self-organization into a torus-like configuration, where $\Omega$ is constant along field lines, winding stops, and the magnetorotational instability is avoided (Duez et al. 2006). In numerical simulations of protoneutron stars, it is standard to find that the magnetic backreaction saturates at $\lesssim 1 \%$ of the mechanical shear stress (Cook et al. 2003; Duez et al. 2006). Whether core super-rotation also drives a turbulent, self-sustaining, hydromagnetic dynamo in the face of strong stratification is less clear. Shear-driven turbulence in a stratified spherical shell automatically supplies the non-zero helicity which a dynamo requires (Gellert et al. 2011), and the magnetic Prandtl number easily exceeds the dynamo threshold $\sim 10^{2} E^{3 / 4}$ in a neutron star. However, the interplay between $S$ and $\sigma$ is not understood fully; recent work suggests that the dynamo depends weakly on $S$ and generates a predominantly dipolar field except for Ro $\sim 1$, but the jury is still out (Šimkanin \& Hejda 2011). 11 Two potentially observable manifestations are suggested by terrestrial analogies: geomagnetic jerks, wherein small changes in the length of the day are communicated to the magnetic field via a Hartmann current system connecting the Ekman/buoyancy layer to the interior (Davis 1994; Davis \& Whaler 1997), and longitudinal drift of equatorial geomagnetic spots, driven by zonal flows over $\sim 10^{7}$ rotation periods (Stanley \& Mohammadi 2008; Šimkanin \& Hejda 2011). 12 Spot or pole drift occurs over roughly the same time-scale as the magnetospheric switching observed in objects like PSR B1828-11 (Lyne et al. 2010).

Secondly, core super-rotation opens up new channels of gravitational-wave emission (van Eysden \& Melatos 2008; Bennett et al. 2010). The free energy in the sheared flow can excite nonaxisymmetric instabilities, e.g. the herringbone, Taylor-Görtler, and Stewartson

11 Braithwaite (2006) demonstrated simultaneously the twin phenomena of self-limiting toroidal field generation and a self-sustaining dynamo loop within a unified, self-consistent simulation of a differentially rotating, nonconvective star with and without stable stratification. Tayler and magnetorotational instabilities disrupt the toroidal field well before it grows to equipartition, provided that the magnetic diffusion lengthscale is shorter than the buoyancy length-scale (always true in a neutron star). Counter-intuitively, in the stratified system, a self-sustaining dynamo establishes itself more easily, the toroidal field builds up to saturation more slowly, and at saturation the toroidal field fluctuates less. In a statistically steady state, the magnetic back-reaction saturates at $\approx 5 \%$ of the average magnetic pressure, much less than the mechanical stress driving the differential rotation.

12 Loper (1971) argued that magnetic and buoyancy forces can conspire to mimic unstratified Ekman pumping under special circumstances, clouding the picture further. Jiefu (1995) agreed but showed that the quasi-steady, sheared flow in the interior persists much longer than $t_{E}$. 
layer modes familiar from Newtonian and superfluid spherical Couette flow (Nakabavashi et al. 2002; Hollerbach 2003; Peralta et al. 2006a; Peralta \& Melatos 2009) and their stratified counterparts (Smirnov et al. 2005; Munro et al. 2010). 13 14 The theoretical machinery exists to treat such instabilities in a neutron star context, including stratification, compressibility, magnetic fields, and a differentially rotating background in general (Mendell 2001; Glampedakis \& Andersson 2006), but it has only been applied to r-modes on a uniformly rotating background at the time of writing. 15 Core super-rotation also complicates gravitational-wave detection: the gravitational-wave phase may drift relative to the rotational phase inferred from radio pulse timing. Finally, as the Reynolds number $E^{-1}$ is large, the interior flow is likely to be turbulent, even when $S$ is large (Greenstein 1970; Melatos \& Peralta 2007, 2010). Stratified turbulence collapses down to two dimensions (i.e. spherical shells) only when the activity parameter $\operatorname{Ro}^{3} S^{-1} E^{-1}$ drops below $\approx 7$, which condition is avoided for Ro $\gtrsim 10^{-2}$, i.e. throughout much of Figure 1 [see Iida et al. (2009) and $\S 2$ in Melatos \& Peralta (2010)]. Stochastic gravitational radiation from hydrodynamic turbulence imposes a fundamental noise floor on gravitational-wave observations of fossil rotators with Ro $\gg 1$ even with the current generation of long-baseline interferometers (Melatos \& Peralta 2010).

In summary, stratification limits Ekman pumping to a thin buoyancy layer in a neutron star, leaving a super-rotating core as the crust brakes electromagnetically. The crust-core shear grows, until dissipation (viscous or Eddington-Sweet) kicks in, then diminishes. Of the radio pulsars discovered to date, a handful of young objects (some Crab-like, some magnetarlike) are predicted to have unexpectedly high Rossby numbers Ro $\gtrsim 10^{-1}$, i.e. their cores are a fossil record of their angular velocities at birth. It is realistic to think that more candidate fossils will be discovered by future surveys. Moreover, there are 24 objects with Ro $\geq 10^{-2}$ already known, some dominated by viscous diffusion, others by thermally driven circulation, which should be examined as a group for common features. If core super-rotation is confirmed, it will prompt a review of traditional ideas concerning how magnetic fields and gravitational waves are generated by neutron stars.

This research was supported by a CSIRO-University of Melbourne Collaborative Grant.

\footnotetext{
13 For example, Peralta \& Melatos (2009) found Ro $\gtrsim 4 E^{0.4}$ from numerical simulations for superfluid Stewartson layer instabilities, which is satisfied throughout much of Figure 1 .

14 Nonaxisymmetric flows excited in the core at birth should also persist up to the dissipation time-scale $\min \left(t_{\nu}, t_{\kappa}\right)$ (Bennett et al. 2010).

15 Stratification is unimportant for r-mode damping, because r-mode circulation is nearly nonradial, unlike spin down. Compressibility roughly doubles the r-mode damping time (Glampedakis \& Andersson 2006).
} 
I thank R. Hoey, K. Poon, and C. Peralta for discussions on numerical simulations of stratified flows, M. Bennett and C. van Eysden for discussions on stratified Ekman pumping in gravitational-wave applications and glitch recovery, and D. Yakovlev and R. Sawyer for references concerning thermodynamic and transport coefficients in bulk nuclear matter.

\section{REFERENCES}

Abney M., Epstein R. I., 1996, Journal of Fluid Mechanics, 312, 327

Alford M. G., Schmitt A., Rajagopal K., Schäfer T., 2008, Reviews of Modern Physics, 80, 1455

Alpar M. A., Chau H. F., Cheng K. S., Pines D., 1996, ApJ, 459, 706

Alpar M. A., Langer S. A., Sauls J. A., 1984, ApJ, 282, 533

Andersson N., Sidery T., Comer G. L., 2007, MNRAS, 381, 747

Avogadro P., Barranco F., Broglia R. A., Vigezzi E., 2007, Phys. Rev. C, 75, 012805

Babaev E., 2009, Physical Review Letters, 103, 231101

Baiko D. A., Haensel P., Yakovlev D. G., 2001, A\&A, 374, 151

Bastrukov S. I., Papoyan V. V., Podgainyi D. V., 1999, Astrophysics, 42, 177

Baym G., Lamb D. Q., Lamb F. K., 1976, ApJ, 208, 829

Bennett M. F., van Eysden C. A., Melatos A., 2010, MNRAS, pp 1370-+

Braithwaite J., 2006, A\&A, 449, 451

Braithwaite J., Nordlund A., 2006, A\&A, 450, 1077

Clark A., 1973, Journal of Fluid Mechanics, 60, 561

Clark A., Clark P. A., Thomas J. H., Lee N.-H., 1971, Journal of Fluid Mechanics, 45, 131

Cook J. N., Shapiro S. L., Stephens B. C., 2003, ApJ, 599, 1272

Cutler C., Lindblom L., 1987, ApJ, 314, 234

Davis R. G., 1994, Geophys. Res. Lett., 21, 1815 
Davis R. G., Whaler K. A., 1997, Physics of the Earth and Planetary Interiors, 103, 181

Duck P., Foster M., 2001, Annual Review of Fluid Mechanics, 33, 231

Duez M. D., Liu Y. T., Shapiro S. L., Shibata M., Stephens B. C., 2006, Phys. Rev. D, 73, 104015

Easson I., 1979a, ApJ, 233, 711

Easson I., 1979b, ApJ, 228, 257

Friedlander S., 1976, Journal of Fluid Mechanics, 76, 209

Gellert M., Rüdiger G., Hollerbach R., 2011, MNRAS, 414, 2696

Glampedakis K., Andersson N., 2006, MNRAS, 371, 1311

Glampedakis K., Andersson N., Samuelsson L., 2011, MNRAS, 410, 805

Goedbloed J. P. H., Poedts S., 2004, Principles of Magnetohydrodynamics

Gotthelf E. V., Vasisht G., Boylan-Kolchin M., Torii K., 2000, ApJ, 542, L37

Greenspan H. P., Howard L. N., 1963, Journal of Fluid Mechanics, 17, 385

Greenstein G., 1970, Nature, 227, 791

Gudmundsson E. H., Pethick C. J., Epstein R. I., 1982, ApJ, 259, L19

Guillot T., 1999, Planet. Space Sci., 47, 1183

Haskell B., Pizzochero P., Sidery T., 2011, ArXiv e-prints

Haskell B., Pizzochero P. M., Sidery T., 2012, MNRAS, 420, 658

Hibschman J. A., Arons J., 2001, ApJ, 554, 624

Hollerbach R., 2003, Journal of Fluid Mechanics, 492, 289

Hollerbach R., 2009, Physics Letters A, 373, 3775

Iida O., Tsuzuki N., Nagano Y., 2009, Theoretical and Computational Fluid Dynamics, 23, 109

Jahan-Miri M., 2002, MNRAS, 330, 279

Jiefu M., 1995, Geophysical and Astrophysical Fluid Dynamics, 81, 159 
Jones P. B., 1991, ApJ, 373, 208

Jones P. B., 2006, MNRAS, 371, 1327

Kaiser N., Brockmann R., Weise W., 1997, Nuclear Physics A, 625, 758

Kinney J. B., Mendell G., 2003, Phys. Rev. D, 67, 024032

Kitiashvili I., Gusev A., 2008a, Advances in Space Research, 42, 1391

Kitiashvili I. N., Gusev A. V., 2008b, Astronomy Reports, 52, 61

Konar S., Bhattacharya D., 1997, MNRAS, 284, 311

Lander S. K., Andersson N., Glampedakis K., 2012, MNRAS, 419, 732

Lander S. K., Jones D. I., Passamonti A., 2010, MNRAS, 405, 318

Link B., 2012, MNRAS, 421, 2682

Livingstone M. A., Kaspi V. M., Gotthelf E. V., Kuiper L., 2006, ApJ, 647, 1286

Loper D. E., 1971, Physics of the Earth and Planetary Interiors, 4, 129

Lyne A., Hobbs G., Kramer M., Stairs I., Stappers B., 2010, Science, 329, 408

Melatos A., Peralta C., 2007, ApJ, 662, L99

Melatos A., Peralta C., 2010, ApJ, 709, 77

Melatos A., Warszawski L., 2009, ApJ, 700, 1524

Mendell G., 1998, MNRAS, 296, 903

Mendell G., 2001, Phys. Rev. D, 64, 044009

Munro R. J., Foster M. R., Davies P. A., 2010, Physics of Fluids, 22, 054108

Nakabayashi K., Tsuchida Y., Zheng Z., 2002, Physics of Fluids, 14, 3963

Page D., 1998, in N. Shibazaki ed., Neutron Stars and Pulsars: Thirty Years after the Discovery Thermal Evolution of Isolated Neutron Stars. pp 183-+

Payne D. J. B., Melatos A., 2004, MNRAS, 351, 569

Peralta C., Melatos A., 2009, ApJ, 701, L75 
Peralta C., Melatos A., Giacobello M., Ooi A., 2005, ApJ, 635, 1224

Peralta C., Melatos A., Giacobello M., Ooi A., 2006a, ApJ, 644, L53

Peralta C., Melatos A., Giacobello M., Ooi A., 2006b, ApJ, 651, 1079

Reisenegger A., Goldreich P., 1992, ApJ, 395, 240

Ruderman M., Zhu T., Chen K., 1998, ApJ, 492, 267

Rüdiger G., Shalybkov D. A., Schultz M., Mond M., 2009, Astronomische Nachrichten, 330, 12

Sakurai T., Clark A., Clark P. A., 1971, Journal of Fluid Mechanics, 49, 753

Saz Parkinson P. M., 2010, ApJ, 725, 571

Seveso S., Pizzochero P. M., Haskell B., 2012, ArXiv e-prints

Shemar S. L., Lyne A. G., 1996, MNRAS, 282, 677

Shternin P. S., Yakovlev D. G., 2008, Phys. Rev. D, 78, 063006

Sidery T., Alpar M. A., 2009, MNRAS, 400, 1859

Sidery T., Passamonti A., Andersson N., 2010, MNRAS, 405, 1061

Smirnov S. A., Boyer D. L., Baines P. G., 2005, Physics of Fluids, 17, 086601

Song X., Richards P. G., 1996, Nature, 382, 221

Spence G. S. M., Foster M. R., Davies P. A., 1992, Journal of Fluid Mechanics, 243, 33

St-Maurice J.-P., Veronis G., 1975, Journal of Fluid Mechanics, 68, 417

Stairs I. H., Lyne A. G., Shemar S. L., 2000, Nature, 406, 484

Stanley S., Mohammadi A., 2008, Physics of the Earth and Planetary Interiors, 168, 179

Strohmayer T. E., Watts A. L., 2006, ApJ, 653, 593

Torrieri G., Mishustin I., 2010, Phys. Rev. C, 82, 055202

Tsakadze J. S., Tsakadze S. J., 1980, Journal of Low Temperature Physics, 39, 649

Urpin V., 2003, A\&A, 408, 331 
Šimkanin J., Hejda P., 2011, Geophysical Journal International, 185, 637

van Eysden C. A., Melatos A., 2008, Classical and Quantum Gravity, 25, 225020

van Eysden C. A., Melatos A., 2010, MNRAS, 409, 1253

van Eysden C. A., Melatos A., 2011, Journal of Low Temperature Physics, 165, 1

van Eysden C. A., Melatos A., 2012, Journal of Low Temperature Physics, 166, 151

Walin G., 1969, Journal of Fluid Mechanics, 36, 289

Warszawski L., Melatos A., 2008, MNRAS, 390, 175

Warszawski L., Melatos A., 2011, MNRAS, 415, 1611

Waszek L., Irving J., Deuss A., 2011, Nature Geoscience, 4, 264

Weber F., 2005, Progress in Particle and Nuclear Physics, 54, 193

Wong T., Backer D. C., Lyne A. G., 2001, ApJ, 548, 447

Yakovlev D. G., Levenfish K. P., Shibanov Y. A., 1999, Physics Uspekhi, 42, 737

Yuan J. P., Manchester R. N., Wang N., Zhou X., Liu Z. Y., Gao Z. F., 2010, ApJ, 719, L111

Zhang J., Song X., Li Y., Richards P. G., Sun X., Waldhauser F., 2005, Science, 309, 1357 